\title{
Gallic acid suppresses inflammation in dextran sodium sulfate-induced colitis in mice: possible mechanisms
}

\begin{abstract}
Inflammatory bowel diseases (IBD) encompass at least two forms of intestinal inflammation: Crohn's disease and ulcerative colitis (UC). Both conditions are chronic and inflammatory disorders in the gastrointestinal tract, with an increasing prevalence being associated with the industrialization of nations and in developing countries. Patients with these disorders are 10 to 20 times more likely to develop cancer of the colon. The aim of this study was to characterize the effects of a naturally occurring polyphenol, gallic acid (GA), in an experimental murine model of UC. A significant blunting of weight loss and clinical symptoms was observed in dextran sodium sulfate (DSS)-exposed, GA-treated mice compared with control mice. This effect was associated with a remarkable amelioration of the disruption of the colonic architecture, a significant reduction in colonic myeloperoxidase (MPO) activity, and a decrease in the expression of inflammatory mediators, such as inducible nitric oxide synthase (iNOS), cyclooxygenase (COX)-2, and pro-inflammatory cytokines. In addition, GA reduced the activation and nuclear accumulation of p-STAT3Y705, preventing the degradation of the inhibitory protein $\mathrm{I} \kappa \mathrm{B}$ and inhibiting of the nuclear translocation of $\mathrm{p} 65-\mathrm{NF}-\kappa \mathrm{B}$ in colonic mucosa. These findings suggest that GA exerts potentially clinically useful anti-inflammatory effects mediated through the suppression of p65-NF- $\mathrm{BB}$ and IL-6/p-STAT3Y705 activation.
\end{abstract}

Keyword: Gallic acid; Inflammatory bowel disease; NF-кB; STAT3; Ulcerative colitis 